\title{
Preparation and characterization of albumin-heparin microspheres
}

\author{
H.F.M. Cremers, G. Kwon*, Y.H. Bae*, S.W. Kim, R. Verrijk ${ }^{\dagger}$, H.P.J.M. \\ Noteborn ${ }^{\dagger}$ and J. Feijen \\ Department of Chemical Technology, University of Twente, PO Box 217, 7500 AE Enschede, The Netherlands; \\ *Department of Pharmaceutics \& Center for Controlled Chemical Delivery, University of Utah, Salt Lake City, UT \\ 84108, USA; ${ }^{\dagger}$ Division of Experimental Therapy, The Netherlands Cancer Institute, Amsterdam, The Netherlands

\begin{abstract}
Albumin-heparin microspheres were prepared by a two-step process which involved the preparation of a soluble albumin-heparin conjugate, followed by formation of microspheres from this conjugate or by a double cross-linking technique involving both coupling of soluble albumin and heparin and microsphere stabilization in one step. The first technique was superior since it allowed better control over the composition and the homogeneity of the microspheres. Microspheres could be prepared with a diameter of 5-35 $\mu \mathrm{m}$. The size could be controlled by adjusting the emulsification conditions. The degree of swelling of the microspheres was sensitive to external stimuli, and increased with increasing $\mathrm{pH}$ and decreasing ionic strength of the medium.
\end{abstract}

Keywords: Microspheres, ion-exchange, hydroge/s, drug delivery

Received 22 April 1993; accepted 22 June 1993

Conventional administration of drugs using simple drug dosage forms like tablets, capsules and bolus injections is not always optimal for effective treatment. In the last decade numerous drug delivery systems have been developed, giving better control over drug release rates and drug targeting. Among these novel drug delivery systems, microspheres take an important place. Microspheres have been prepared from a variety of synthetic and natural polymers ${ }^{1-4}$.

Albumin microspheres (AMS) have seen an extensive development during the past 20 years. AMS, loaded with cytotoxic agents such as adriamycin, have been widely investigated as possible chemoembolization devices $^{5,6}$. These AMS are generally prepared by the formation of a water-in-oil emulsion and subsequent stabilization of the protein droplets by using chemical cross-linking agents such as glutaraldehyde, formaldehyde or 2,3-butadione, or by thermal denaturation of the protein. Problems encountered in the preparation of drug-loaded AMS are mainly related to the hydrophobicity of the AMS, and drug loading and release properties. Drug loading has to be done during the microsphere preparation process and chemical reactions with or thermal decomposition of the drug cannot always be prevented ${ }^{7,8}$. The in vitro drug release is generally considered to be biphasic. During the first phase a large amount of the drug may be released within a few minutes to hours. This initial large and fast release (i.e. the burst effect) is followed by a much slower, first-order release ${ }^{9}$.

Correspondence to Dr J. Feijen.
Albumin has an iso-electric point of $5.3^{10}$ and under physiological conditions, AMS exert some ionexchange properties, but these properties are too weak to be of interest. By the introduction of polyanions, however, the ion-exchange properties are increased and some of the disadvantages described above can be overcome. Microspheres prepared from albumin and polyanions are more hydrophilic and, together with the ion-exchange properties, this enables drug loading of ionic drugs after the preparation process. Previously, Longo et $a l^{7}$ have prepared ion-exchange albumin microspheres by incorporating poly $(\alpha-\mathrm{L}-$ glutamic acid) in the microspheres. Willmott et al. ${ }^{11}$ prepared microspheres from albumin and poly(aspartic acid).

In this paper the preparation and characterization of biodegradable ion-exchange microspheres containing albumin and heparin are described. These microspheres can be prepared by techniques similar to those used in the preparation of AMS. Previously, we have described a method ${ }^{12}$ starting with a preformed albumin-heparin conjugate $^{13}$ and stabilization with glutaraldehyde (twostep procedure) giving albumin-heparin conjugate microspheres (AHCMS). This method was evaluated more thoroughly, with regard to the influence of the cross-linking conditions on the swelling behaviour. Furthermore, another method is described in which the conjugate preparation step is omitted. These albumin-heparin microspheres (AHMS) can be prepared using a double cross-linking technique by stabilization of an aqueous mixture of albumin and heparin with 1-ethyl-3-(3-dimethylaminopropyl)carbodiimide (EDC) followed by glutaraldehyde. 


\section{MATERIALS AND METHODS}

\section{Materials}

Human serum albumin was obtained from the Central Laboratory of the Dutch Red Cross Blood Transfusion Service, Amsterdam, The Netherlands. Heparin from porcine intestinal mucosa, with a specific activity of $165 \mathrm{IU} / \mathrm{mg}$ as indicated by the manufacturers, was obtained from Diosynth (Oss, The Netherlands). Cibracon Blue Sepharose (CBS) and Diethylaminoethyl (DEAE)-Sepharose 4B were obtained from Pharmacia Fine Chemicals (Uppsala, Sweden). Highly refined olive oil, porcine albumin (fraction V) and glutaraldehyde ( $25 \%$ aqueous solution, grade I) were obtained from Sigma (St Louis, MO, USA). Glutaraldehyde (25\% aqueous solution) was obtained from Merck (Darmstadt, Germany). Albumin-heparin conjugates, prepared from porcine albumin with heparin contents of $5.9 \%$ (p-AHC1), $16.0 \%$ (p-AHC2) and $21.5 \%$ (pAHC 3 ), as indicated by the manufacturer, were obtained from Holland Biomaterials Group (Enschede, The Nellerlands). Phosphate buffered saline (PBS) was obtained from the NPBI (Emmer Compascuum, The Netherlands). All other reagents were obtained, in the highest possible grade, from Merck (Darmstadt, Germany).

\section{Glutaraldehyde purification}

As a result of aldol condensation reactions, commercially available glutaraldehyde solutions usually contain $\alpha, \beta$-unsaturated dimers, trimers or higher oligomers $^{14}$. To remove these impurities, glutaraldehyde solutions were purified by distillation at $13 \mathrm{~mm}$ $\mathrm{Hg}$, as described by Gillett and $\mathrm{Gull}^{15}$. The fraction distilling at $70-75^{\circ} \mathrm{C}$ was collected. The distilled glutaraldehyde was dissolved in boiling water to obtain 1 . 2.5, 5 and $25 \% \mathrm{w} / \mathrm{v}$ aqueous solutions. The purified solutions were stored at $4^{\circ} \mathrm{C}$. The UV spectrum of glutaraldehyde shows an absorption peak at $280 \mathrm{~nm}$, whereas the condensation products strongly absorb at $235 \mathrm{~nm}$. Glutaraldehyde solutions were only used if the $A_{235}: A_{280}$ ratio was lower than 0.4 .

\section{Synthesis, purification and characterization of soluble conjugates of albumin and heparin}

The synthesis of the albumin-heparin conjugate was carried out by a slightly modified method of Hennink et al ${ }^{13}$. Figure $1 a$ shows a schematic representation of the conjugate synthesis and purification procedure. $\Lambda$ lbumin $(12.5 \mathrm{~g})$ and heparin $(3.75 \mathrm{~g})$ were dissolved in $175 \mathrm{ml}$ of water. This solution was adjusted to $\mathrm{pH}$ 5.0 with $\mathrm{HCl}(0.2 \mathrm{M}$ ). EDC (a solution of $1.24 \mathrm{~g}$ in $8 \mathrm{ml}$ of water) was added dropwise to the reaction mixture over a period of $4 \mathrm{~h}$ at room temperature. Thirty minutes after the addition of EDC, the $\mathrm{pH}$ was adjusted to 7.6 with $\mathrm{NaOH}(1 \mathrm{M})$ and the mixture was gently stirred for $20 \mathrm{~h}$ at $4 \% \mathrm{C}$. Finally the solution was dialysed twice against $0.025 \mathrm{M}$ Tris- $\mathrm{HCl}$ buffer, $\mathrm{pH} 7.5$, using cellulose Visking ${ }^{\mathrm{B}}$ membranes.

Unreacted heparin was removed from the reaction mixture using a CBS column. The column was equilibrated using CBS-equilibration buffer $(0.17 \mathrm{M} \mathrm{NaCl} /$ $0.025 \mathrm{M}$ Tris-HCl, $\mathrm{pH} 7.5$ ). This buffer was also used to dissolve and load the conjugate/heparin/albumin mixture and to elute the heparin. The bound material (the conjugate and unreacted albumin) was eluted using CBS-elution buffer ( $1 \mathrm{M} \mathrm{KSCN/0.025} \mathrm{M} \mathrm{Tris-HCl,}$

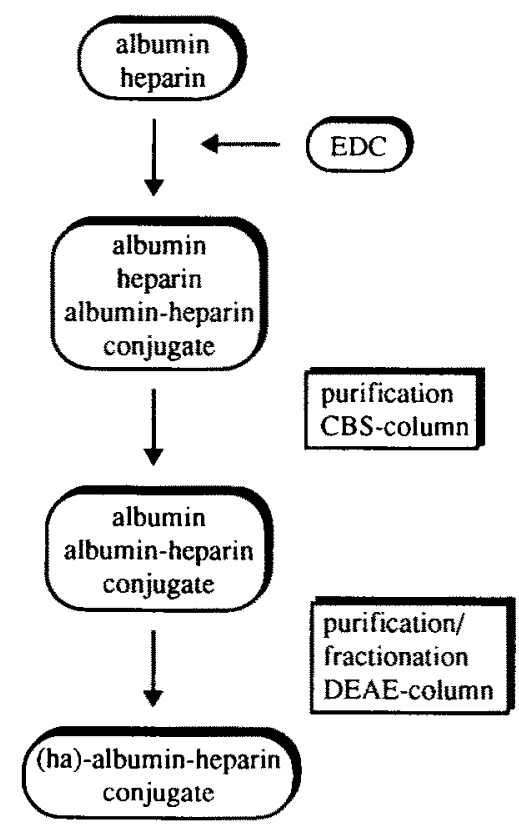

a

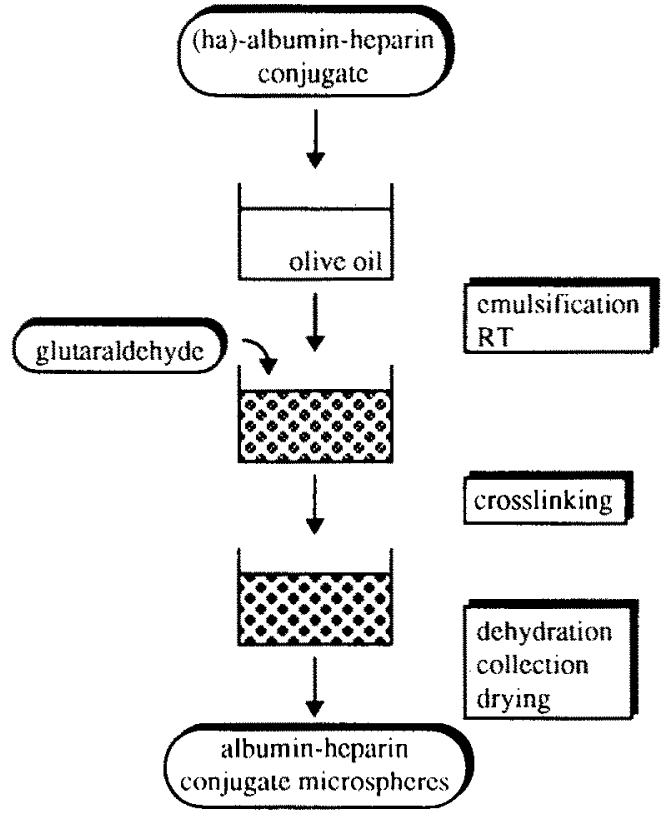

$\boldsymbol{b}$

Figure 1 Schematic representation of AHCMS preparation process: a, first step: AHC synthesis and purification/fractionation; b, second step: microsphere preparation from AHC using chemical stabilization with glutaraldehyde. 
$\mathrm{pH}$ 7.5). The conductivity of the eluent was monitored using a conductivity cell (Philips, PW9513, cell constant $=1.66$; Philips, Eindhoven, The Netherlands) connected to a conductivity meter (Philips PW9527 digital conductivity meter; Philips). The protein content of the eluent was monitored on line using a UV-monitor (Single Path Monitor UV-1, Optical Unit and Control Unit; Pharmacia). Both monitors were connccted to a double channel recorder (BD41; Kipp en Zonen, Delft, The Netherlands). Flow rates were controlled using a peristaltic pump (2132 Microperpex Peristaltic Pump; LKB, Uppsala, Sweden). After this purification step the eluted material was dialysed extensively against water and freeze dried.

The unreacted albumin was removed using a DEAE column. The column was equilibrated with DEAEequilibration buffer $(0.3 \mathrm{M} \mathrm{NaCl} / 0.025 \mathrm{M}$ Tris- $\mathrm{HCl}, \mathrm{pH}$ 7.0). The albumin/conjugate mixture was dissolved and loaded in $0.025 \mathrm{M}$ Tris-HCl, $\mathrm{pH}$ 7.0. Unreacted albumin was eluted using DEAE-equilibration buffer. Unfractionated purified conjugate (AHC) was obtained by elution with DEAE-elution buffer-I $(0.9 \mathrm{M} \mathrm{NaCl} /$ $0.025 \mathrm{M}$ Tris-HCl, pH 7.0). After this purification step the AHC was dialysed extensively against water using cellulose Visking " membranes and treeze dried.

To obtain high affinity conjugate (ha-AHC) the DEAE purification step was carried out using two elution buffers ${ }^{16}$. Loosely bound conjugate (low affinity conjugate) was first eluted using a buffer with a low ionic strength: DEAE-elution buffer-II $10.6 \mathrm{M} \mathrm{NaCl} /$ $0.025 \mathrm{M}$ Tris-HCl, pH 7.0). Subsequently, the ha-AHC was eluted using DEAE-elution buffer-I.

The heparin content of the conjugate was determined using the metachromic assay described by Jacques and Wollin ${ }^{17}$, as modified by Hennink et $a l^{13}$. The albumin content was determined using two methods, by UV/vis spectroscopy at $280 \mathrm{~nm}$ and by a modified biuret method ${ }^{18}$.

\section{Microsphere preparation: two-step procedure}

Chemically stabilized AHCMS were prepared according to methods previously described for $\mathrm{AMS}^{19}$. This method for preparing the microspheres is outlined in Figure $1 b$.

To a flat-bottomed glass beaker (diameter $60 \mathrm{~mm}$, height $110 \mathrm{~mm}$ ) equipped with four baffles (depth $4 \mathrm{~mm}$ ) positioned against the wall of the beaker was added $125 \mathrm{ml}$ of olive oil. A four-bladed axial-flow impeller, motor-driven using an IKA-RW20DZM mechanical stirrer (Janke \& Kunkel, Staufen, Germany), was placed in the centre of the beaker with a $3 \mathrm{~mm}$ distance between the baffles and the impeller blade. The impeller was submerged two thirds into the oil. After pre-stirring for $30 \mathrm{~min}$ at 850 r.p.m., $0.6 \mathrm{ml}$ of a conjugate solution in PBS $(100 \mathrm{mg} / 0.6 \mathrm{ml}$ PBS) was added dropwise from a syringe to the olive oil. After emulsification by stirring for an additional period of $15 \mathrm{~min}, 0.15 \mathrm{ml}$ of a glutaraldehyde solution of the desired concentration was added. The emulsion was then stirred at 850 r.p.m. for $1 \mathrm{~h}$ to allow cross-linking of the albumin moieties. Subsequently $50 \mathrm{ml}$ of acetone was added and the emulsion was stirred for a further $10 \mathrm{~min}$. The microspheres were isolated by centrifugation for $15 \mathrm{~min}$ at 2700 r.p.m., followed by decanting of the supernatant. The microspheres were then washed several times with acetone and finally dried in vacuo at $50^{\circ} \mathrm{C}$.

To study the effect of (1) cross-link density, (2) reduction of the imines initially formed by the reaction of an aldehyde group with a primary amine group and (3) quenching of the unreacted aldehyde groups, several reaction conditions were varied. Cross-link density was varied by using glutaraldehyde solutions ranging from 1.0 to $25.0 \% \mathrm{w} / \mathrm{v}$. Imines were reduced by the addition of $20 \mu \mathrm{l}$ of an $\mathrm{NaCNBH}_{3}$ solution $(5 \mathrm{M})$ to the emulsion, prior to the addition of the glutaraldehyde solution. Quenched AHCMS (AHCMSQ) were prepared by the addition of $100 \mu \mathrm{l}$ of a $10 \% \mathrm{w} / \mathrm{v}$ aqueous glycine solution to the emulsion after crosslinking with glutaraldehyde. After addition of this solution, the emulsion was stirred for a further $1 \mathrm{~h}$.

Thermally stabilized microspheres (AHCMSmw) were prepared by heating the AHC solution/olive oil emulsion in a microwave oven (Toshiba ER 7700DW; Toshiba, Tokyo, Japan). After $15 \mathrm{~min}$ of emulsification as described above, the emulsion was equally divided into three $60 \mathrm{ml}$ glass bottles and placed in a microwave oven. The bottles were heated in three cycles $(2 \mathrm{~min}-1 \mathrm{~min}-1 \mathrm{~min})$ at full power $(650 \mathrm{~W})$. Between the cycles, the bottles were shaken to homogenize the emulsion. After microwave treatment, the bottles were left to cool to room temperature. Then AHCMSmw were washed, dehydrated and dried as described above. After collection, the microspheres were washed to remove unbound material by ultrasonic resuspension in $5 \mathrm{ml}$ of ethanol. PBS $(45 \mathrm{ml})$ was added and the suspension was rotated for $30 \mathrm{~min}$. After centrifugation and decanlation of the washing medium, $50 \mathrm{ml}$ of fresh PBS were added and the microspheres were washed once more. This procedure was repeated twice with distilled water. Microspheres were dehydrated with acetone and dried as described above.

\section{Microsphere preparation: double cross-linking technique}

AHMS were also prepared from free albumin and heparin, using a double cross-linking technique (Figure 2). In these experiments, the same equipment was used as in the two-step procedure. A solution $(0.4 \mathrm{ml})$ of $100 \mathrm{mg}$ of albumin and $50 \mathrm{mg}$ of heparin in $0.6 \mathrm{ml}$ of water was added dropwise to $125 \mathrm{ml}$ olive vil, pre-slirred for $30 \mathrm{~min}$ at 850 r.p.m. After emulsification for $15 \mathrm{~min}$ at 850 r.p.m., $0.1 \mathrm{ml}$ of $20 \% \mathrm{w} / \mathrm{v}$ EDC solution was added to couple the heparin to the albumin. After stirring for $4 \mathrm{~h}, 0.1 \mathrm{ml}$ of an aqueous glutaraldehyde solution $(25 \% \mathrm{w} / \mathrm{v})$ was added for additional stabilization by cross-linking the albumin. The emulsion was stirred for a further $1 \mathrm{~h}$. Microspheres were collected, washed and dried as described above.

\section{Particle size distribution of the microspheres}

The determination of the size and size distribution of the microspheres $(n>250)$ was carried out using a 


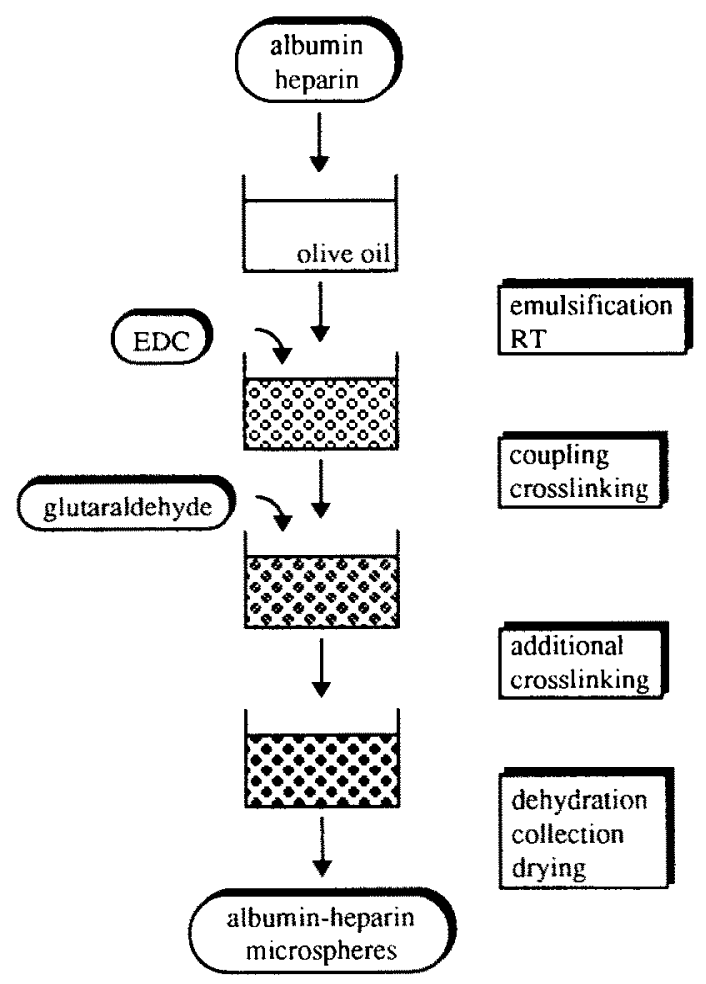

Figure 2 Schematic representation of AHMS preparation process: a solution of albumin and heparin is emulsified in olive oil, then EDC is added to couple albumin and heparin, and subsequently glutaraldehyde is added to stabilize the microspheres.

scanning electron microscope (SEM) (JEOL JSM-35CF scanning electron microscope; JEOL, Tokyo, Japan) operated at $15 \mathrm{kV}$. For this purpose, a microsphere suspension in ethanol was dried on an aluminium chip and coated with a conducting gold layer using a Balzers Union Sputtering Device (Cathode Sputtering Unit 07 120; Balzers, Liechtenstein). Size distributions were also determined using a light microscope (LM) (Ortolux 2 POL-BK; Leitz, Wetzlar, Germany) fitted with a camera (Wild MPS52; Wild-Leitz, Heerburg, Switzerland) and a calibrated graticule. The SEM and LM micrographs were analysed using a particle size analyser (Carl-Zeiss, TG3).

\section{Swelling experiments}

The swelling behaviour of the microspheres was evaluated by measuring the mean diameter $(\bar{d}=$ $\left.\Sigma n_{i} d_{i} / \Sigma n_{i}\right)$ and the size distribution of the spheres (using two different techniques) in phosphate and citrate buffers of varying $\mathrm{pH}$ and ionic strength.

In the first protocol, light microscopy was used to determine the size distribution of the microspheres. Microspheres (1 mg) were resuspended ultrasonically in $0.5 \mathrm{ml}$ of ethanol. From this suspension, 10 samples of $25 \mu$ were pipetted into separate wclls of a 96 -well microtitre plate. The ethanol was evaporated overnight, and subsequently $0.1 \mathrm{ml}$ of buffer was added to the wells and the microspheres were resuspended ultrasonically in the buffers. Light microscopy pictures were taken, and the mean diameter and the size distribution of the microspheres was determined by image analysis techniques using a Quantimet 520 Plus morphometer (Leica, Rijswijk, The Netherlands).

Swelling was also studied using a flow cytometer (FACStar $^{+}$, Beckton-Dickinson, Mountain View, CA, USA). Microspheres (11 mg) were resuspended ultrasonically in $1.1 \mathrm{ml}$ of ethanol. From this suspension $0.1 \mathrm{ml}$ was pipetted into counting vials. To each counting vial, $0.9 \mathrm{ml}$ of buffer was added. To remove the ethanol from these solutions, the vials were centrifuged, the supernatant was decanted and fresh buffer was added. The microspheres were resuspended in this buffer by vortexing. The mean diameter and the size distribution of the microspheres were determined using the FACStar ${ }^{+}$. Per sample, 10000 microspheres were counted.

\section{RESULTS AND DISCUSSION}

AHCMS were prepared from a prefabricated albuminheparin conjugate by an emulsion stabilization technique. Since endogenous materials are used it is expected that the microspheres may be biocompatible as well as biodegradable. Due to the introduction of strong anionic groups by heparin incorporation, the microspheres will exert ion-exchange properties.

Commercial glutaraldehyde solutions usually contain $\alpha, \beta$-unsaturated oligomers and polymers. The presence of these compounds can be detected using UV spectroscopy. Glutaraldehyde gives an absorption peak at $280 \mathrm{~nm}$, whereas the $\alpha, \beta$-unsaturated oligomers and polymers strongly absorb at $235 \mathrm{~nm}$. The impurities can be removed from the glutaraldehyde solutions by distillation or by multiple extraction with activated charcoal. Since distillation and subsequent dilution gives better control over the concentration of the final solution, this method was used.

Figure 3 shows the UV spectra of two commercially available glutaraldehyde solutions and a purified solution. The high grade product, which is obtained in sealed ampoules under a nitrogen atmosphere, showed a small peak at $235 \mathrm{~nm}$. The other commercial product, however, showed a much larger and broader peak in this region. After distillation of this product, this peak

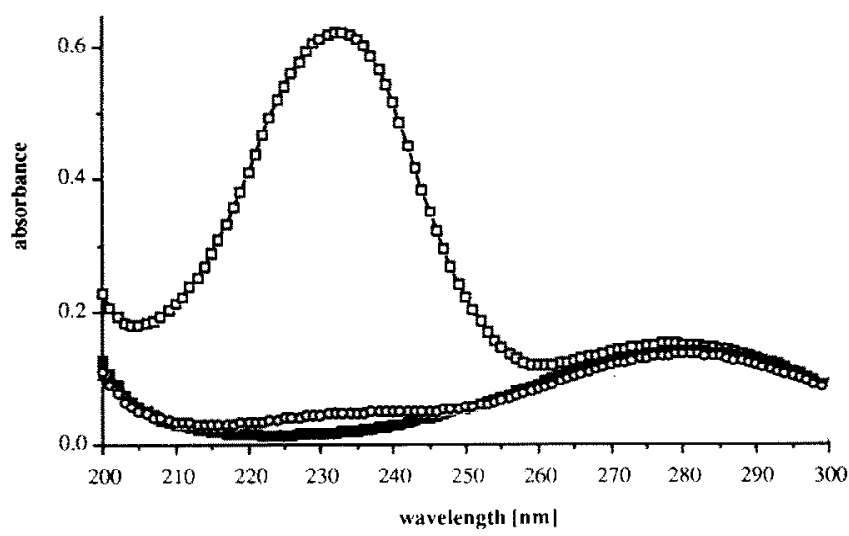

Figure 3 UV spectra of several glutaraldehyde solutions. 0 . Commercial $25 \%$ glutaraldehyde solution, Grade I, obtained in sealed ampoules under $\mathrm{N}_{2}$ atmosphere; $\square$. commercial $25 \%$ glutaraldehyde solution, normal grade; D. previous product after distillation $\left(70-75^{\circ} \mathrm{C}, 13 \mathrm{~mm} \mathrm{Hg}\right)$. 
had disappeared completely, indicating removal of the $\alpha, \beta$-unsaturated oligomers and polymers. After storage at $4^{\circ} \mathrm{C}$ for 2.5 years, some impurities had formed again. The solution, however, was used regularly during this period, undergoing warming up to room temperature. Solutions, continuously stored at $4^{\circ} \mathrm{C}$, did not show any changes in the UV spectrum during this period. This is in agreement with the results of Gillett and Gull $^{15}$, who showed that the formation of these impurities is mainly determined by the storage temperature. Oxygen atmosphere and light did not seem to have any effect. Although the impurity of the normal grade commercial product seems substantial it should be taken into account that the polymer, absorbing at $235 \mathrm{~nm}$, has a much higher extinction coefficient. Commercial products rarely contain more than $1 \%$ of the polymeric impurity ${ }^{20}$.

After purification using CBS and DEAE-Sepharose column chromatography, the conjugate of albumin and heparin (AHC), containing $10.7 \pm 0.9 \% \quad \mathrm{w} / \mathrm{w}$ of heparin, was prepared in a yield of about $50 \%$. This is in good agreement with results obtained by Hennink et $a l^{13}$. Fractionation of the conjugate was achieved using three different buffers during the DEAE purification step. The elution profile from the DEAE column is shown in Figure 4. The first two peaks refer to weakly bound material, mainly albumin, which was eluted using the DEAE equilibration buffer. The presence of two peaks is caused by the temporarily lower ionic strength due to the loading of the material onto the column using a buffer of lower ionic strength. This is confirmed by the dip in the conductivity curve. Using DEAE-elution buffer-II, loosely bound (low affinity) AHC was eluted (third peak). Subsequently, high affinity conjugate was eluted using DEAE-elution buffer-I (fourth peak). This procedure allowed the preparation of high affinity conjugate with a heparin content of $10.2 \pm 1.2 \% \mathrm{w} / \mathrm{w}$ in a yield of $35 \%$ based on the initial amount of albumin.

Microspheres of albumin and heparin were prepared

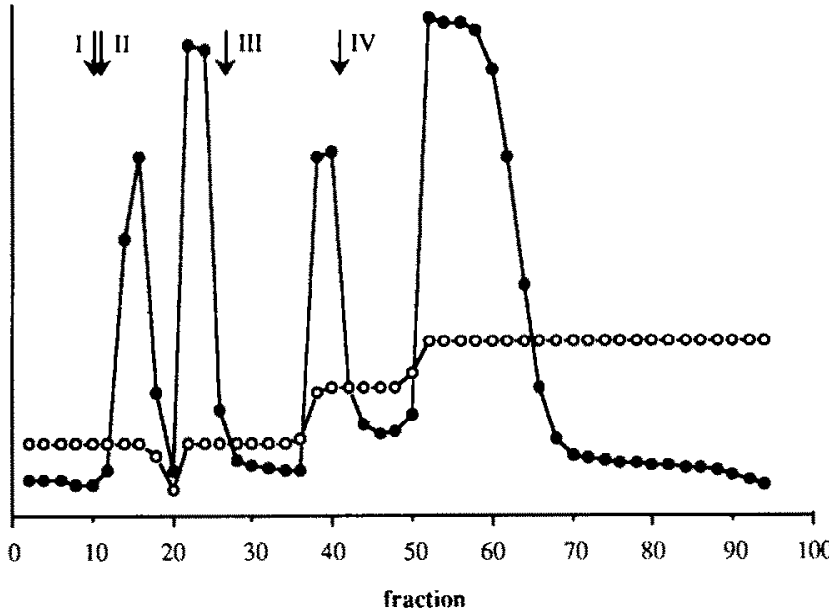

Figure 4 Elution profile on DEAE-Sepharose 4B. Initial buffer: $0.3 \mathrm{M} \mathrm{NaCl} / 0.025 \mathrm{M}$ Tris-HCl, $\mathrm{pH} 7.0$; 1: conjugate/ albumin solution in $0.025 \mathrm{M}$ Tris- $\mathrm{HCl}, \mathrm{pH} 7.0 ; 11: 0.3 \mathrm{M} \mathrm{NaCl}$, $0.025 \mathrm{M}$ Tris $-\mathrm{HCl}, \mathrm{pH} 7.0 ;$ III: $0.6 \mathrm{M} \mathrm{NaCl}, 0.025 \mathrm{M}$ Tris $-\mathrm{HCl}, \mathrm{pH}$ $7.0 ; \mathrm{IV}: 0.9 \mathrm{M} \mathrm{NaCl}, 0.025 \mathrm{M}$ Tris- $\mathrm{HCl}, \mathrm{pH} 7.0: \mathrm{O}$, conductivity; absorbance.

by the emulsion stabilization method. Under the applied emulsification conditions the diameter of glutaraldehyde cross-linked AHCMS was approximately $5-35 \mu \mathrm{m}$. Cross-link density (i.e. the concentration of the glutaraldehyde solution), reduction and quenching did not change the size distribution significantly (Table 1).

Figure 5 shows SEM micrographs of the AHCMS05, of the ha-AHCMS50 and of the AHMS (prepared by the double cross-linking technique). From these micrographs it can be seen that AHCMS05 and AHMS had approximately the same size. AHMS, however, had a much more ruffled surface. This is indicative of matrix inhomogeneity which can be caused by a 'phase separation' of the polymer solution, favouring the presence of albumin at the oil-water interface,

Table 1 Microsphere preparation conditions and results

\begin{tabular}{|c|c|c|c|c|c|c|c|}
\hline \multirow[t]{2}{*}{ Code } & \multirow{2}{*}{$\begin{array}{l}\text { Material } \\
\text { heparin content } \\
(\%)\end{array}$} & \multirow{2}{*}{$\begin{array}{l}\text { Stirring } \\
\text { speed } \\
\text { (r.p.m.) }\end{array}$} & \multicolumn{3}{|l|}{ Stabilization } & \multirow{2}{*}{$\begin{array}{l}\text { Size* } \\
(\mu \mathrm{m})\end{array}$} & \multirow{2}{*}{$\begin{array}{l}\text { Yield }{ }^{\dagger} \\
(\%)\end{array}$} \\
\hline & & & $\begin{array}{l}\text { Glutaraldehyde } \\
(\%)\end{array}$ & Reduction & Quenching & & \\
\hline $\begin{array}{l}\text { AHCMSO2 } \\
\text { AHCMSO5 } \\
\text { AHCMS10 } \\
\text { AHCMS10R } \\
\text { AHCMS10Q } \\
\text { AHCMS10RQ }\end{array}$ & $\begin{array}{l}10.7 \\
10.7 \\
10.7 \\
10.7 \\
10.7 \\
10.7\end{array}$ & $\begin{array}{l}850 \\
850 \\
850 \\
850 \\
850 \\
850\end{array}$ & $\begin{array}{l}0.2 \\
0.5 \\
1.0 \\
0.2 \\
1.0 \\
1.0\end{array}$ & $\begin{array}{l}- \\
- \\
- \\
+ \\
+\end{array}$ & $\begin{array}{l}- \\
- \\
- \\
+ \\
+\end{array}$ & $\begin{array}{l}4-36 \\
5-35 \\
7-35 \\
5-37 \\
5-35 \\
5-35\end{array}$ & $\begin{array}{l}75 \\
72 \\
79 \\
73 \\
69 \\
75\end{array}$ \\
\hline $\begin{array}{l}\text { ha-AHCMS50 } \\
\text { p-AMS } \\
\text { p-AHC1MS } \\
\text { p-AHC2MS } \\
\text { p-AHC } \\
\text { AHCMS } \\
\text { AHMS** }\end{array}$ & $\begin{array}{r}10.2 \\
0.0 \\
5.9 \\
16.0 \\
21.5 \\
10.7 \\
33.3\end{array}$ & $\begin{array}{r}1230 \\
950 \\
950 \\
950 \\
950 \\
850 \\
850\end{array}$ & $\begin{array}{l}5.0 \\
0.5 \\
0.5 \\
0.5 \\
0.5 \\
\text { thermal } \\
\text { EDC/5.0\% }\end{array}$ & $\begin{array}{l}- \\
- \\
- \\
- \\
-\end{array}$ & $\begin{array}{l}- \\
- \\
- \\
- \\
-\end{array}$ & $\begin{array}{r}12-80 \\
2-18 \\
2-27 \\
2-27 \\
3-60 \\
5-40 \\
4-30\end{array}$ & $\begin{array}{l}76 \\
73 \\
78 \\
79 \\
78 \\
76 \\
50\end{array}$ \\
\hline
\end{tabular}

- As determined from LM and SEM micrographs.

Based on the initial amount of conjugate or albumin plus heparin.

Microspheres prepared from the high affinity atbumin-heparin conjugate fraction eluted from DEAE column.

Conjugates obtained irom HBG, Enschede, The Netherlands, prepared from porcine albumin. p-AMS were prepared from porcine albumin obtained from Sigma.

Microspheres prepared by thermal stabilization; stirring speed refers to initial emulsification conditions.

*Microspheres prepared using the double cross-tinking technique; heparin content refers to initial ratio of albumin and heparin in the solution 

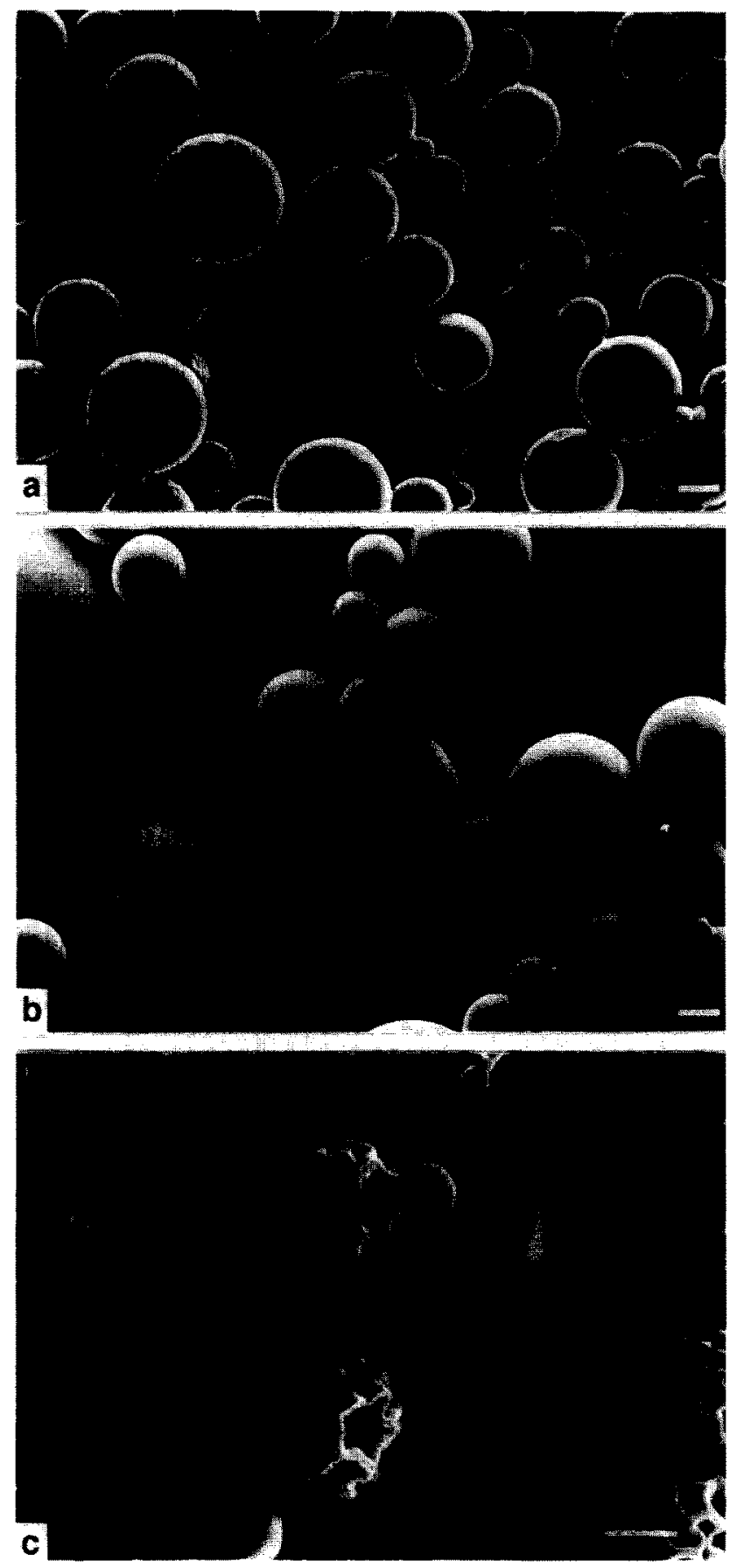

Figure 5 SEM micrographs of microspheres prepared from albumin and heparin: a, AHCMSO5, prepared from AHC, cross-linked with $0.5 \%$ glutaraldehyde; b, ha-AHCMS50, prepared from high-affinity $\mathrm{AHC}$, cross-linked with $5.0 \%$ glutaraldehyde; c, AHMS, prepared from albumin and heparin using the double cross-linking technique. Bar indicates $10 \mu \mathrm{m}$.

resulting in an albumin-poor phase at the core of the droplet and an albumin-rich phase at the outside. This eventually results in a tightly cross-linked outer layer of the microspheres and a loosely stabilized core. During the washing procedure, the core material is removed and the microspheres collapse. Consequently the yield after washing of the AHMS was much lower than the yields obtained when the microspheres were prepared from the conjugates (Table 1).
Micrograph analysis of the microspheres showed that the size distribution of the microspheres was influenced by the kind of conjugate used. Using the high affinity conjugate a more viscous aqueous solution was obtained. Assuming that the high affinity conjugate has more charged groups than the unfraction* ated conjugate, which would account for the high affinity towards the DEAE-Sepharose, the increased viscosity is a phenomenon often observed with polyelectrolyte solutions, which generally become more viscous with increasing degree of dissociation. However, the difference between high affinity conjugate and unfractionated conjugate may also be an increased mobility and chain length of the heparin moieties of the high affinity conjugate which in turn also would account for the increased viscosity of solutions of this material. In order to obtain microspheres of approximalely the same dianeter as microspheres prepared from an unfractionated conjugate, a higher stirring speed was applied. The size distribution determined, however, was broader: $12-80 \mu \mathrm{m}$. The influence of the viscosity of the aqueous phase on the size distribution of the spheres is also shown in Figures 6 and 7.

Figure 6 shows the cumulative size distribution of pAHCMS. With increasing heparin content, i.e. with increasing viscosity of the aqueous phase, the $\mathrm{p}^{\text {- }}$ AHCMS became larger and the size distribution became broader. Figure 7 shows the size distributions of AHCMS prepared under different reaction conditions. Increasing the conjugate concentration of the aqueous phase resulted in larger microspheres. Figure 7 also shows the influence of increasing the amount of the aqueous phase.

As already mentioned, the size of the microspheres increased with increasing amount of the aqueous phase. Burger et al. ${ }^{19}$ showed that the diameter of AMS increased with increasing viscosity of the aqueous phase as well as with decreasing stirring speed, decreasing viscosity of the organic phase and increasing amount of aqueous phase. A number of essentially

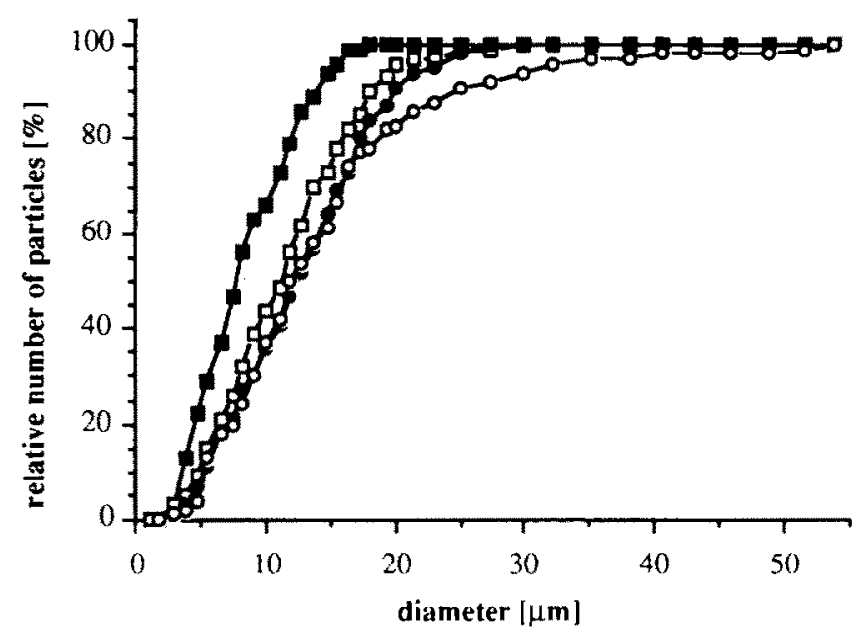

Figure 6 Cumulative size distributions of microspheres $(n>250)$ prepared from porcine albumin or porcine AHC with increasing heparin contents:, p-AMS, $\square, p$ AHC1MS, 5.9\% heparin; 0 -AHC2MS, $16.0 \%$ heparin; $O$, p-AHC3MS, $21.5 \%$ heparin. 


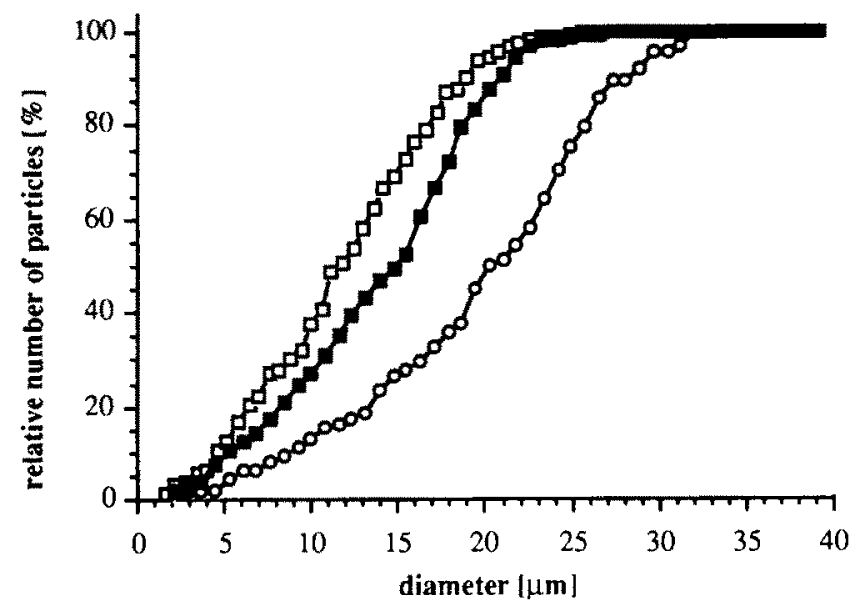

Figure 7 Cumulative size distributions of AHCMS $(n>250): \square, 100 \mathrm{mg} \mathrm{AHC}$ dissolved in $0.6 \mathrm{ml}$ of aqueous phase, $0.4 \mathrm{ml}$ injected in olive oll; $\square, 100 \mathrm{mg}$ AHC dissolved in $0.6 \mathrm{ml}$ of aqueous phase, $0.6 \mathrm{ml}$ injected in olive oil; $O$, $100 \mathrm{mg} \mathrm{AHC}$ dissolved in $0.4 \mathrm{ml}$ of aqueous phase, $0.4 \mathrm{ml}$ injected in olive oil.

similar empirical relationships between average particle size and these parameters have been described in the literature on suspension polymerization. Equation 1 provides a qualitative guide for controlling the size of microspheres produced by that method.

$$
\bar{d} \propto K \frac{D_{\mathrm{v}} R v_{\mathrm{d} \gamma}}{D_{\mathrm{s}} N v_{\mathrm{m}} C_{\mathrm{s}}}
$$

where $\bar{d}$ is the average droplet (particle) size; $K$ symbolizes parameters such as vessel design and type of stirrer; $D_{\mathrm{v}}$ is the diameter of the vessel; $D_{\mathrm{s}}$ is the diameter of the stirrer; $R$ is the volume ratio of the droplet phase to suspension medium; $N$ is the stirring speed; $v_{d}$ is the viscosity of the droplet phase; $v_{m}$ is the viscosity of the suspension medium; $\gamma$ is the surface tension between the two immiscible phases; and $C_{s}$ is the stabilizer concentration ${ }^{21}$.

The colour of the microspheres was influenced by the glutaraldehyde concentration used to stabilize the microspheres. In the case of $1.0 \%$ glutaraldehyde cross-linking, the microspheres were yellow-brown. This colour became darker with increasing glutaraldehyde concentration. Thermally stabilized microspheres as well as microspheres stabilized with $0.2 \%$ glutaraldehyde were almost colourless. The colour of the microspheres was also influenced by reduction of the imine with $\mathrm{NaCNBH}_{3}$ during cross-linking. Microspheres cross-linked with $1.0 \%$ glutaraldehyde in the presence of this reducing agent had a colourless instead of a brown appearance. Quenching with glycine did not affect the colour. The colouring of the microspheres can be explained by the reaclions involved in the cross-linking with glutaraldehyde. Although the exact cross-linking mechanism is not yet fully understood, it is generally accepted that the initial step is the formation of an imine by the reaction of an aldehyde group with a primary $\varepsilon$-amine group of a lysine residue ${ }^{22,23}$. In the presence of a reducing agent like $\mathrm{NaCNBH}_{3}$, this group is readily converted to a stable secondary amine. Subsequently, the formation of a second amine may be followed by an intramolecular ring closing reaction. The resulting six membered imine is readily reduced by $\mathrm{NaCNBH}_{3}$ to give a piperidine ring ${ }^{24}$.

In the absence of the reducing agent, however, numerous subsequent reactions may occur, with the initial imine acting as an intermediate. These reactions may result in several types of oligomeric and polymeric cross-links, the most important being pyridinium compounds formed by oxidative cyclization of the imine ${ }^{25-27}$. Some of the reaction products may account for the brown colour ${ }^{28}$. Although polymeric cross-links consisting of up to 40 glutaraldehyde molecules have been described, it is more likely that only oligomeric cross-links are formed. Recently, Olde Damink $^{29}$ has shown that an average of three glutaraldehyde molecules was consumed per reacted amine group in the cross-linking of collagen.

In the case of thermal stabilization, different types of cross-links are formed. In the microwave oven, the oil was heated to $120^{\circ} \mathrm{C}$. At these temperatures structural changes in the albumin are irreversible and stable microspheres are obtained by formation of intermolecular disulphide bridges between free cysteine $\mathrm{SH}$ groups on adjoining protein chains ${ }^{2,30}$. Sokoloski and Royer $^{22}$ also report the formation of $N$-(oL-2-amino-2carboxy-ethyl)-L-lysine (lysinoalanine) cross-links; however, these authors do not explain the reaction mechanism of the formation of these cross-links.

Compared with AMS, AHCMS are easily suspended in aqueous media. This may be explained by the way the particles are prepared. Widder et al. ${ }^{31}$ hypo hesize that the hydrophobicity of AMS is due to the apolar regions of the albumin aligning at the oil-water interface to form a hydrophobic crust. AHCMS, however, contain polyanionic heparin chains which may be exposed at the surface of the particles, increasing the hydrophilicity.

ESCA studies of freeze dried AHCMS, data presented in Table 2, showed that sulphur was present at the surface, that the sodium content was increased and that the carbon:oxygen ratio was decreased, implying that heparin is present at the surface of microspheres when contacted with water. Furthermore, AHCMS had a $\zeta$ potential of $-40.0 \mathrm{mV}$ as compared to $-17 \mathrm{mV}$ for AMS, which is also consistent with the presence of heparin entities at the surface ${ }^{12}$.

The LM micrographs shown in Figure 8 demonstrate qualitatively the influence of the cross-link density

Table 2 ESCA data for AMS and AHCMS*

\begin{tabular}{lllr}
\hline Sample & Element & $\begin{array}{l}\text { Binding } \\
\text { energy }(\mathrm{eV})\end{array}$ & Atom \% \\
\hline AMS & $\mathrm{C}(1 \mathrm{~s})$ & 282 & 71.6 \\
& $\mathrm{~N}(1 \mathrm{~s})$ & 396 & 8.9 \\
& $\mathrm{O}(1 \mathrm{~s})$ & 528 & 17.4 \\
& $\mathrm{Na}(2 \mathrm{~s})$ & 61 & 1.7 \\
AHCMS & $\mathrm{C}(1 \mathrm{~s})$ & 282 & 63.8 \\
(freeze dried) & $\mathrm{N}(1 \mathrm{~s})$ & 396 & 8.1 \\
& $\mathrm{O}(1 \mathrm{~s})$ & 528 & 19.9 \\
& $\mathrm{~S}(2 \mathrm{p})$ & 168 & 0.6 \\
& $\mathrm{Na}(2 \mathrm{~s})$ & 61 & 2.4 \\
\hline
\end{tabular}

Data obtained trom Kwon et al. ${ }^{2}$ 


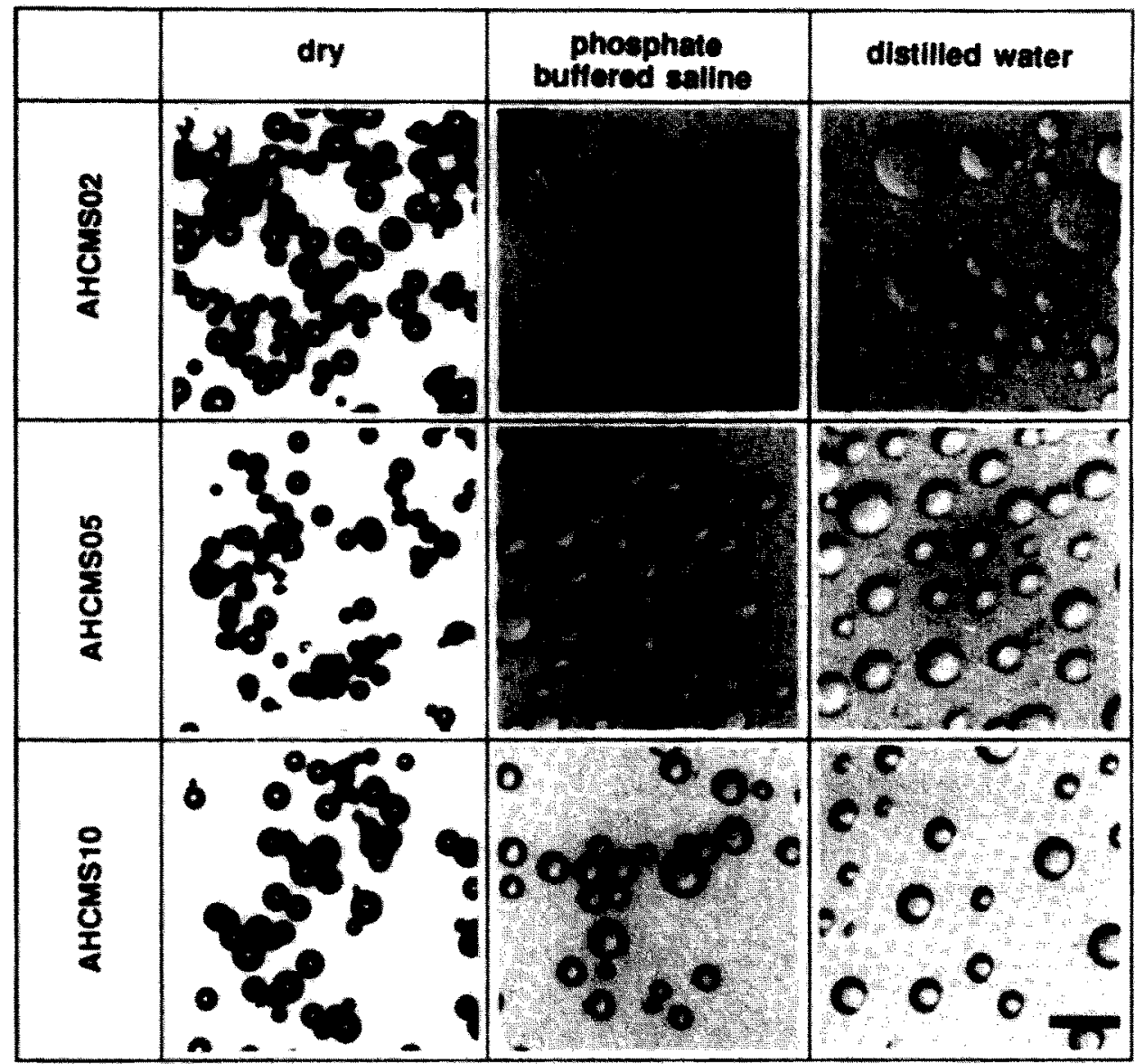

Figure 8 LM micrographs of AHCMS02, AHCMS05 and AHCMS10 in the dry state, swollen in PBS at pH 7.4 and $I=0.17$, and in distilled water. Bar indicates $50 \mu \mathrm{m}$.

and the medium on the swelling behaviour of AHCMS. The LM experiments were not suitable for determination of quantitative effects of the cross-link density of the particles and of the $\mathrm{pH}$ and ionic strength of the swelling medium. This was due to the small number of particles counted in these experiments (below 80). However, it can be seen that the degree of swelling in distilled water was higher than in PBS. Figure 8 also demonstrates that the degree of swelling decreased with increasing glutaraldehyde cross-linking.
Quantitative swelling data were obtained from the flow cytometric measurements. In these experiments 10000 microspheres were counted to give size distributions as shown in Figures 9 and 11. Figure 9 gives the size distribution of AHCMSO5 as a function of the $\mathrm{pH}$ at an ionic strength of 0.4 . With increasing $\mathrm{pH}$, the size distribution became broader and shifted to higher values. This is also shown in Figure 10. The $\mathrm{pH}$ dependent swelling behaviour of AHCMS can be explained by the ionization of functional groups of the

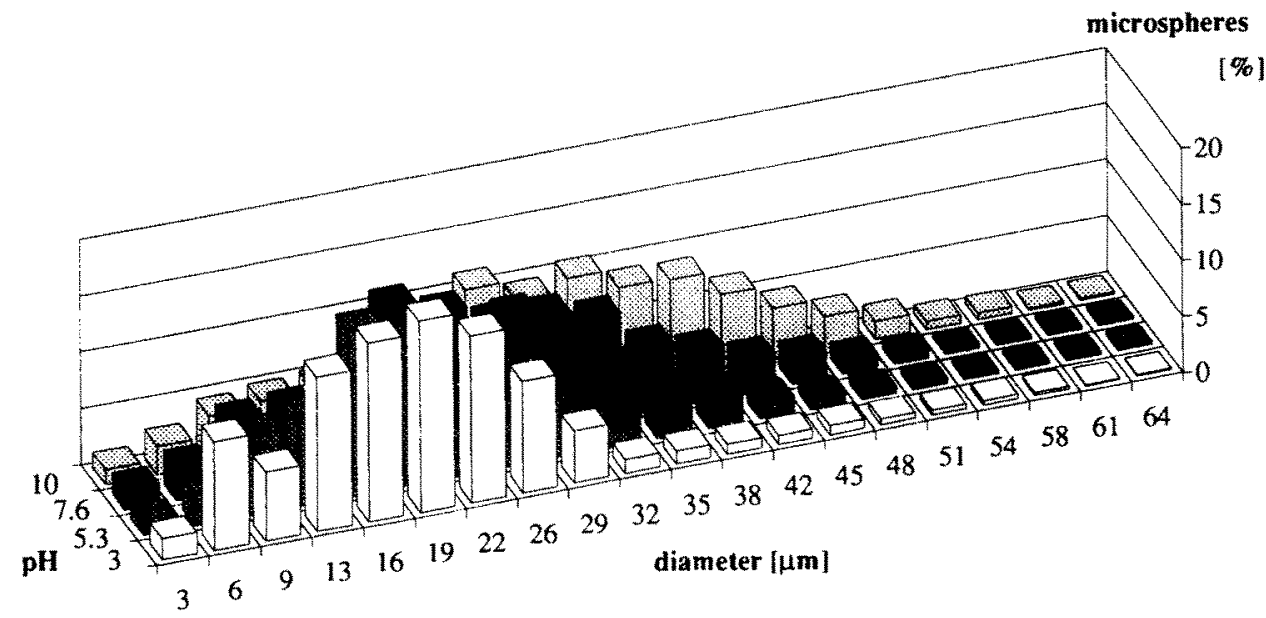

Figure 9 Size distributions of AHCMS05 in buffers of varying $\mathrm{pH}$ at an ionic strength $I=0.4$. Size distributions were determined using a flow cytometer; 10000 particles were counted. 


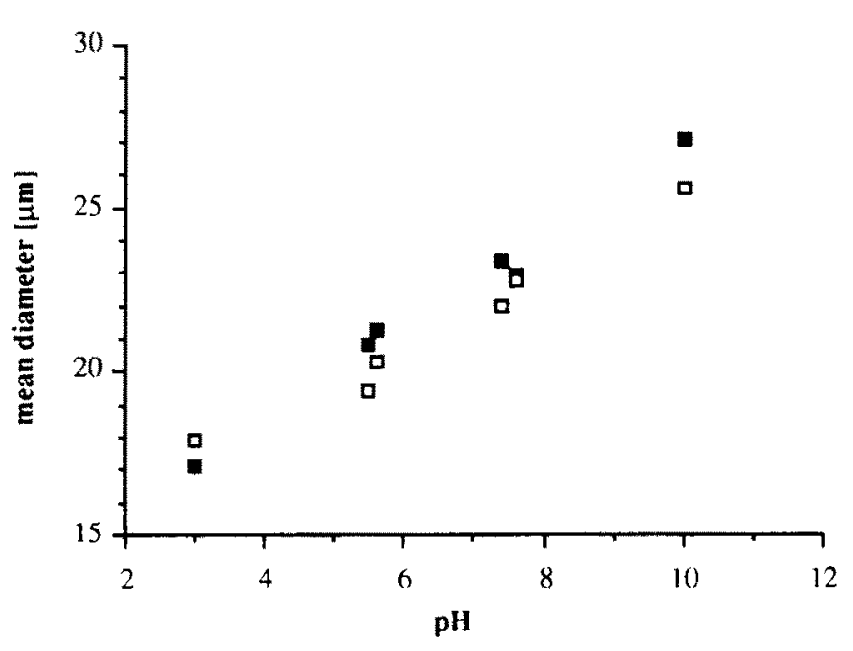

Figure 10 Mean diameter of AHCMS as a function of the $\mathrm{pH}$ at ionic strength, $I=0.4: \square$. AHCMS05; $\square$. AHCMS10. Each point represents the mean diameter of 10000 microspheres, as determined using a flow cytometer.

albumin (Asp, Glu, Lys) and of the heparin (sulphate, sulphamate and carboxylate groups). An increase in negatively charged groups in the matrix leads to increased repulsive forces, resulting in expansion of the network. Such a $p H$-dependent swelling of polyelectrolyte gels has also been observed by Seigel and Firestone ${ }^{32}$.

Counterions in the network may effectively neutralize this repulsion and consequently decrease the swelling. So with increasing ionic strength the swelling should decrease, which is demonstrated in Figures 11 and 12. Figures 10 and 12 also show that the degree of swelling is influenced by the stabilization of the microspheres. At higher cross-link density, swelling is reduced.

The effect of the $\mathrm{NaCNBH}_{3}$ reduction during the cross-linking is shown in Figure 13. This graph shows that the swelling ability of AHCMS10R and AHCMS10RQ is reduced as compared with nonreduced microspheres. This may be caused by the reduction of the initially formed imines, which prevents the formation of oligomeric and polymeric glutaraldehyde cross-links. Another explanation is that in the presence of $\mathrm{NaCNBH}_{3}$, relatively hydrophobic piperidine compounds may be formed ${ }^{24}$, shielding the amino groups, which also results in a decrease in the degree of swelling. Quenching with glycine did not influence the swelling behaviour of the microspheres.

Previously, Kwon et al. ${ }^{33}$ showed that AHCMS can be loaded with high molecular weight compounds by a swelling-deswelling process. The presence of heparin introduces ion-exchange properties, allowing the loading of microspheres with positively charged drugs like adriamycin ${ }^{34}$. These findings suggest that AHCMS can be used as both swelling-controlled and ioncontrolled drug delivery devices.

\section{CONCLUSIONS}

AHCMS can be prepared in a two-stop procodure involving $\mathrm{AHC}$ synthesis and purification in the first step and micrnsphere preparation in the second step. AHMS can be prepared from albumin and heparin using a double cross-linking technique. The latter procedure, however, gives less control over the composition of the microspheres and is therefore not recommended. AHCMS are more hydrophilic than albumin microspheres, due to the presence of the heparin at the surface of the microspheres. This enables the suspension of AHCMS in the absence of surfactants which were necessary to stabilize albumin microsphere suspensions. The stimuli-sensitiveswelling behaviour of the microspheres allows loading of high molecular weight compounds, whereas the ionexchange properties enable the loading of positively charged drugs. In conclusion, due to the improvement of the suspension properties and drug loading characteristics of albumin microspheres by the incorporation of another endogenous material, namely heparin, AHCMS have great potential as drug delivery devices.

\section{ACKNOWLEDGEMENTS}

The authors wish to acknowledge the Netherlands Organization for Scientific Research (NWO) and Theratech Inc., Salt Lake City, Utah for financially

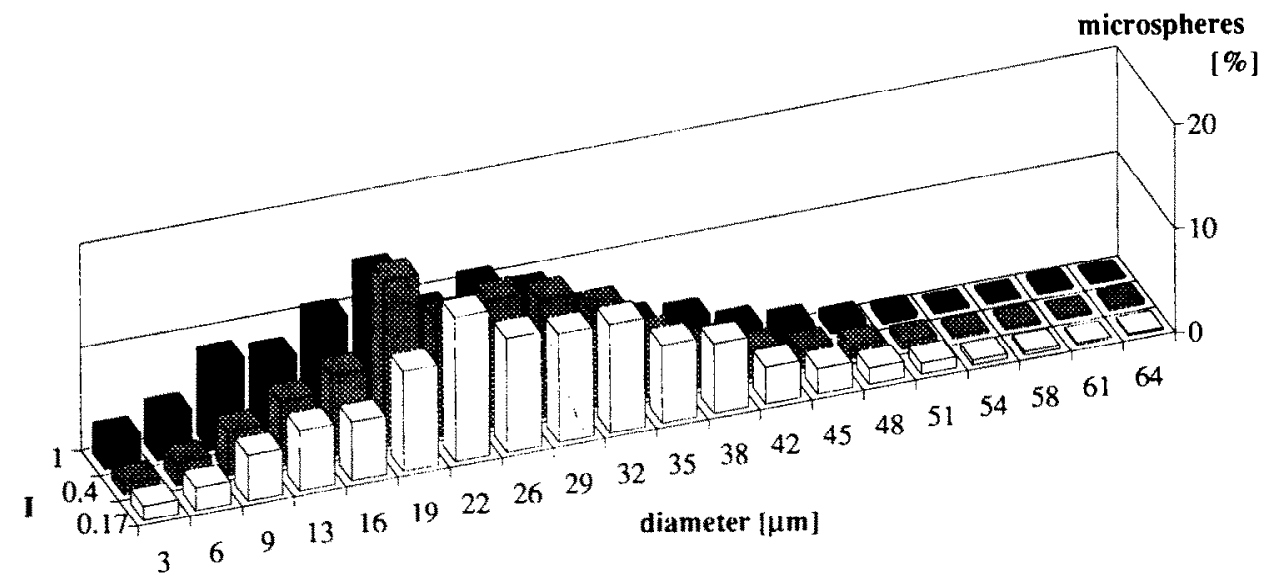

Figure 11 Size distributions of AHCMS05 as a function of the ionic strength at pH 7.4. Size distributions were determined using a flow cytometer; 10000 microspheres were counted. 


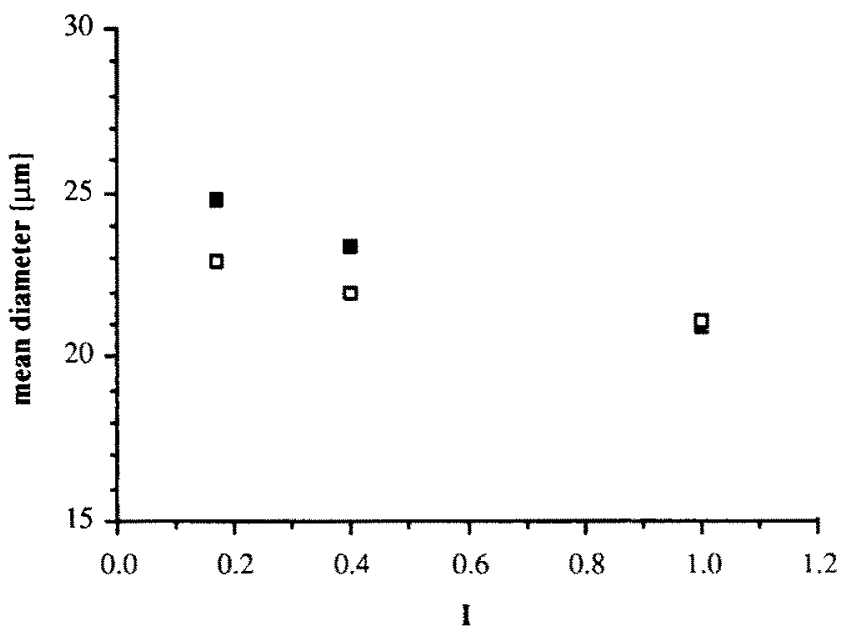

Figure 12 Mean diameter of AHCMS as a function of the ionic strength at $\mathrm{pH} 7.4: \square$, AHCMS05; $\square$, AHCMS10. Each point represents the mean diameter of 10000 microspheres, as determined using a flow cytometer.

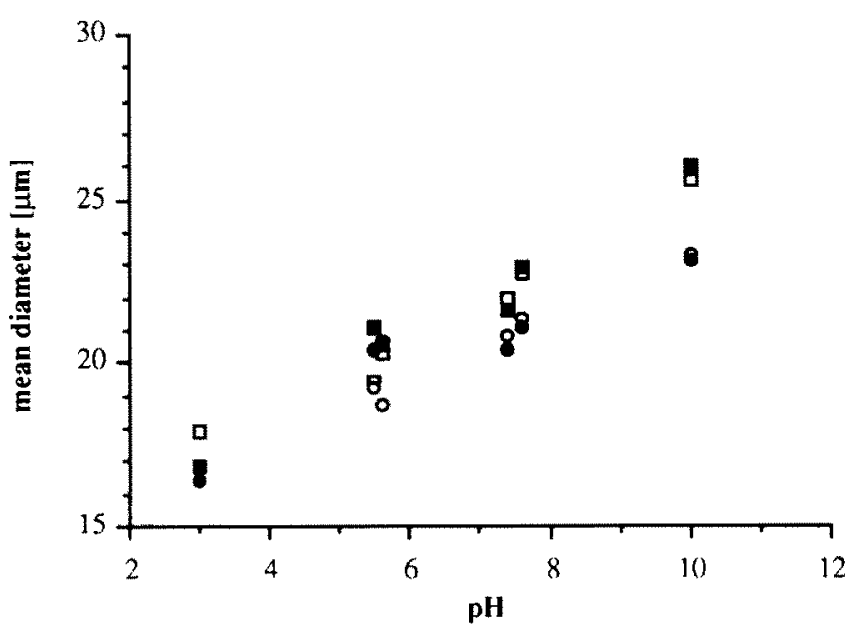

Figure 13 Mean diameter of AHCMS as a function of the $\mathrm{pH}$ at ionic strength, $l=0.4: \square$, microspheres cross-linked with $1.0 \%$ glutaraldehyde; microspheres cross-linked with $1.0 \%$ glutaraldehyde, subsequently quenched with glycine; 0 . microspheres cross-linked with $1 \%$ glutaraldehyde in the presence of $\mathrm{NaCNBH}_{3} ;$, microspheres cross-linked with $1.0 \%$ glutaraldehyde in the presence of $\mathrm{NaCNBH}_{3}$, subsequently quenched with glycine. Each point represents the mean diameter of 10000 microspheres, as determined using a flow cytometer.

supporting this research. Thanks are also due to Jolanda Melching of the University of Twente, to Mark Dessing of the Netherlands Cancer Institute and to Sjef Schakenraad of the University of Groningen for their contributions to the work described in this paper.

\section{REFERENCES}

1 Tomlinson E. Microsphere delivery systems for drug targeting and controlled release. Int I Pharm Tech Prod Mfr 1983; 4: 49-57.

2 Thies C, Bissery M-C. In: Biomedical Applications of Microencapsulation (Ed, F Lim), CRC Press, Boca Raton, FL, 1985: pp 53-74.
3 Wheatley M, Langer, R. Particles as drug delivery sytems. Particulate Sci Technol 1987; 5: 53-64.

4 Davis SS, Illum L. Polymeric microspheres as drug carriers. Biomaterials 1988; 9: 111-115.

5 Widder KJ, Marino PA, Morris RM, Senyei AE. Targeting antineoplastic agents using magnetic albumin microspheres. Polym Biol Med 1983; 2: 201-230.

6 McArdle CS, Lewi H, Hansell D, Kerr DI, McKillop J. Willmott $N$. Cytotoxic-loaded albumin microspheres: a novel approach to regional chemotherapy, Br J Surg 1988; 75: 132-134.

7 Longo WE. Iwata $H$, Lindheimer T, Goldberg EP. Preparation and drug release properties of albuminpolyglutamic acid-adriamycin microspheres. Polym Prepr 1983; 24: 56-57.

8 Law SL, Chiang CH, Lin FM, Teh GW. Effect of stabilization temperature on the degradation of adriamycin in albumin microspheres. Biomater, Art Cells Immob Biotech 1991; 19: 613-629.

9 Tomlinson E, Burger JI, Schoonderwoerd EMA, McVie JG. In: Microspheres and Drug Therapy, Pharmaceutical, Immunological and Mcdical Aspects (Eds SS Davis, L Illum, JG McVie, E Tomlinson), Elsevier, Amsterdam, 1984: pp 75-89.

10 Putnam FW. The Plasma Proteins, Vol 1, 2nd Edn, Academic Press, New York, 1975: pp 133-180.

11 Willmott N, Chen Y, Florence AT. Haemoglobin, transferrin and albumin/polyaspartic acid microspheres as carriers for the cytotoxic drug adriamycin. I. Ultrastructural appearance and drug content. J Controlled Rel 1988; 8: 93-101.

12 Kwoil GS, Bae YH, Kin SW, Crenters H, Feijen J. Preparation and characterization of microspheres of albumin-heparin conjugates. I Colloid Interface Sci 1991; 143: 501-512.

13 Hennink WE, Feijen J, Ebert CD, Kim SW. Covalently bound conjugates of albumin and heparin: synthesis, fractionation and characterization. Thromb Res 1983 29: 1-13.

14 Hardy PM, Nicholls AC, Rydon HN. The nature of glutaraldehyde in aqueous solution. Chem Commun 1969: 565-566.

15 Gillett R, Gull K. Glutaraldehyde-its purity and stability. Histochemie 1972; 30: 162-167.

16 Engbers GHM. The development of heparinized materials with an improved blood compatibility, University of Twente, Enschede. The Netherlands, 1990: pp 71-98

17 Jacques LB, Wollin A. A modified method for the calorimetric determination of heparin. Can J Phys Pharmacol 1967; 45: 787-794.

18 Goa J. A micro biuret method for protein determination Determination of total protein in cerebrospinal fluid. Scand J Clin Lab Invest 1953; 5: 218-222.

19 Burger JJ, Tomlinson E, Mulder EMA, McVie JG. Albumin microspheres for intra-arterial tumour targeting. I. Pharmaceutical aspects, Int I Pharmacol 1985; 23: $333-344$.

20 Korn AH, Feairheller SH, Filachione EM. Glutaraldehyde: nature of the reagent. / Mol Biol 1972; 65: 525529.

21 Arshady R. Albumin microspheres and microcapsules: methodology of manufacturing techniques. / Controlled Rel 1990; 14: 111-131.

22 Sokoloski TV, Royer GP. In: Microspheres and Drug Therapy, Pharmaceutical, Immunological and Medical Aspects (Eds SS Davis, L Illum, JG McVie, E Tomlinson), Elsevier, Amsterdam, 1984: pp 295-307.

23 Habeeb AFSA, Hiramoto R. Reaction of proteins with glutaraldehyde. Arch Biochem Biophys 1968; 126: 1626. 
Borch RF, Bernstein MD, Durst HD. The cyanoborohydrate anion as a selective reducing agent. I Am Chem Soc 1971; 93: 2897-2904.

25 Woodroof EA. Use of glutaraldehyde and formaldehyde to process tissue heart valves. J Bioeng 1978; 2: 1-9.

26 Cheung DT, Nimni ME. Mechanism of crosslinking of proteins by glutaraldehyde I: reaction with model compounds. Connective Tiss Res 1982; 10: 187-199.

27 Johnson TJA. Glutaraldehyde fixation chemistry: oxygen-consuming reactions. Eur J Coll Biol 1987; 45: 160-169.

28 Lubig R, Kusch P, Röper K, Zahn. Zum Reactionsmechanismus von Glutaraldehyd mit Proteinen. Monatsh Chem 1981; 112: 1313-1323.

29 Olde Damink LHH. Structure and properties of crosslinked dermal sheep collagen, University of Twente, Enschede, The Netherlands, 1993: pp 29-54.
30 Wetzel R, Becker M, Behlke J, Billwitz H, Böhm S, Ebert B, Hammann H, Krumbiegel J, Lassmann G. Temperature behaviour of human serum albumin. Eur $J$ Biochem 1980; 104: 469-478.

31 Widder K, Flouret G, Senyei A. Magnetic microspheres synthesis of a novel parental drug carrier. I Pharm Sci 1979; 68: 79-82.

32 Seigel R, Firestone BA. pH-dependent equilibrium swelling properties of hydrophobic polyelectrolyte copolymer gels. Macromolecules 1988; 21: 3254-3259.

33 Kwon GS, Bae YH, Cremers H, Feijen J, Kim SW Release of macromolecules from albumin-heparin microspheres. Int J Pharmacol 1992; 79: 191-198.

34 Cremers HFM, Feijen I, Kwon G, Bae YH, Kim SW, Noteborn IIPJM, McVie IG. Albumin-heparin microspheres as carriers for cytostatic agents. I Controlled Rel 1990; 11: 167-179.

\title{
MATERIALS SCIENCE AND IMPLANT ORTHOPAEDIC SURGERY II
}

\author{
Chandris Hotel Chania, Crete, Greece
}

\author{
June 19 - July 2, 1994
}

The goal of the NATO Advanced Study Institute is to foster understanding and appreciation among surgeons, material scientists, and the manufacturers of implants. This goal is achieved through lectures, discussions, and panels on the clinical aspects of surgery, surface reactivities, wear and ionic leaching debris, implant fixation, healing, immune response, and more.

Lectures will include:

Biomaterials and Implant Surgery, Professor Jack E. Lemons

Clinical Aspects of Dental Implant Surgery, Professor Victor Matukas

Biological Consequences of Ionic Leaching and Debris, Professor Ulrich Gross

Mechanisms of Ionic Leaching and Debris Production, Professor Peter Revell

Science and Engineering of Surface Modifications, Dr Geoff Dearnaley

Cementless and Porous Surface Implants, Dr R.J. Geesink

Aspects of Interfacial Healing, Professor Rik Huiskes

Biochemistry of Bone Adaptation, Professor Lutz Claes

Guided Tissue Regeneration, Professor Thorkild Karring

New Materials and Implant Systems, Professor Klaas de Groot

For further information, please contact: Dr Ram Kossowsky, Director, Material Engineering \& Systerns Division, Emerging Technologies, Inc., 6327 Burchfield Avenue, Pittsburgh, PA 15217, USA. Tel: +14124214408 . Fax: +14124214342 . 